\title{
Neutron beams from deuteron breakup at the 88-Inch cyclotron at Lawrence Berkeley National Laboratory
}

\author{
M.A. McMahann, ,a, L. Ahle ${ }^{2}$, D.L. Bleuel ${ }^{1, b}$, L. Bernstein ${ }^{2}$, B.R. Barquest ${ }^{1}$, J. Cerny ${ }^{1,3}$, L.H. Heilbronn ${ }^{4}$, C.C. Jewett ${ }^{3}$, \\ I. Thompson ${ }^{2}$, and B. Wilson ${ }^{1}$ \\ 1 Nuclear Science Division, Lawrence Berkeley National Laboratory, Berkeley, CA 94720, USA \\ 2 N-Division, Lawrence Livermore National Laboratory, Livermore, CA 94550, USA \\ 3 Department of Chemistry, University of California at Berkeley, Berkeley, CA 94720, USA \\ ${ }^{4}$ Life Science Division, Lawrence Berkeley National Laboratory, Berkeley, CA 94720, USA
}

\begin{abstract}
Accelerator-based neutron sources offer many advantages, in particular tunability of the neutron beam in energy and width to match the needs of the application. Using a recently constructed neutron beam line at the 88 Inch Cyclotron at LBNL, tunable high-intensity sources of quasi-monoenergetic and broad spectrum neutrons from deuteron breakup are under development for a variety of applications.
\end{abstract}

\section{Introduction}

Fast neutrons are finding increasing use for many applications. At the same time, reactor based sources of fast neutrons are disappearing. Accelerator-based sources offer many advantages, not the least of which is the possibility to tune the neutron source in energy and width to match the needs of the application.

Sources of monoenergetic neutrons based on the ${ }^{7} \mathrm{Li}(\mathrm{p}, \mathrm{n})$ reaction [1] are available at accelerator facilities in Europe $[2,3]$, Asia [4], and elsewhere [1,5]. This method - which has been applied over a wide range of energies from a few $\mathrm{MeV}$ through hundreds of $\mathrm{MeV}$ - produces a neutron beam narrow in energy and peaked at the proton beam energy minus the Q-value. Two disadvantages of this method are 1) a broad neutron tail (approximately $40 \%$ of the total neutron flux) at lower energies due to breakup reactions [6] and 2) relatively low cross sections, especially at higher energies. The cross section for this reaction peaks at approximately $6 \mathrm{MeV}$ and decreases rapidly at higher energies [7].

An alternative method to the ${ }^{7} \mathrm{Li}(\mathrm{p}, \mathrm{n})$ reaction for neutron production is deuteron breakup. This mechanism has the potential to produce forward-focused neutrons at higher fluxes, important for cross section measurements on radioactive targets. The cross section for neutrons produced by deuteron breakup increases rapidly above the threshold value of approximately $9 \mathrm{MeV}$ [8]. Typically, as an accelerator-based neutron source, thick, light targets, e.g., $\mathrm{Li}$ or Be, have been utilized $[9,10]$; few measurements of neutron energy spectra produced by deuteron breakup on thin targets are available.

At the 88-Inch Cyclotron at LBNL, a tunable highintensity source of neutrons has been developed that employs deuteron breakup on thin targets. The 88-Inch cyclotron can deliver deuteron beams of $10 \mu \mathrm{A}$ at energies of $\approx 5-65 \mathrm{MeV}$. Energies below $5 \mathrm{MeV}$ are available at lesser intensities. Phase 1 construction of a new beamline for this project is complete

${ }^{a}$ Presenting author, e-mail: p_mcmahan@lbl.gov

${ }^{b}$ Present address: N-Division, LLNL, Livermore, CA 94550, USA and characterization of the neutrons produced in thin target breakup is underway. For applications in which neutron energy is not important, broad spectrum fast neutrons are currently available.

The present studies are aimed at determining the utility of thin target deuteron breakup as a source of quasimonoenergetic neutrons for a variety of applications, by mapping out the phase space of neutron energy, energy width and flux over the full range of initial deuteron energies on several targets.

\subsection{Applications of fast neutrons}

Cross sections for direct neutron reactions - e.g., (n, $\gamma)$, ( $n$, fission), $(n, 2 n)$ - are important for a wide variety of applications that either take place in a neutron environment or employ neutrons as a probe. In the former category are included science-based stockpile stewardship for nuclear weapons programs in a non-testing era, advanced fuel cycle reactors, fusion at high energy densities and s-process nucleosynthesis. In addition to cross sections, the effect of neutrons on materials, on electronics and on humans is of concern in the environment of space, the upper atmosphere, the earth's surface and in special environments such as high energy accelerators and reactors. Examples of applications in which neutrons are employed as a probe are neutron interrogation techniques for detecting special nuclear material, and materials studies using neutron scattering and radiography.

Table 1 summarizes the needs in terms of neutron energy and flux of a sample of applications under consideration at the 88-Inch Cyclotron. The immediate goal of the present work is to determine the phase space of overlap between the capabilities of neutron production at the Cyclotron with the needs of the applications. Because there are tradeoffs between, for example, energy spread and flux for cross section measurements, each experiment will require unique conditions. 
Table 1. Applications of fast neutrons (optimal conditions).

\begin{tabular}{|c|c|c|c|}
\hline Application & $\begin{array}{l}\text { Energy } \\
(\mathrm{MeV})\end{array}$ & $\begin{array}{l}\text { Resolution } \\
(\mathrm{MeV})\end{array}$ & $\begin{array}{l}\text { Flux } \\
\left(\mathrm{n} / \mathrm{cm}^{2} / \mathrm{sec}\right)\end{array}$ \\
\hline \multicolumn{4}{|l|}{ Cross Sections for: } \\
\hline $\begin{array}{l}\text { Weapons } \\
\text { Stewardship [11] } \\
\text { (radioactive targets) }\end{array}$ & $0.1-20$ & $1-2 \mathrm{MeV}$ & $10^{5}-10^{9}$ \\
\hline $\begin{array}{l}\text { Fusion (NIF) [12] } \\
\text { (tertiary diagnostics) }\end{array}$ & $20-30$ & $\begin{array}{l}1-2 \mathrm{MeV} \text { or } \\
\text { integral }\end{array}$ & $10^{5}-10^{6}$ \\
\hline $\begin{array}{l}\text { Advanced Fuel } \\
\text { Cycle: }(n, f)[13]\end{array}$ & $0.5-2$ & Fission-like & $10^{5}-10^{7}$ \\
\hline \multicolumn{4}{|l|}{ Radiation Effects for: } \\
\hline $\begin{array}{l}\text { Electronics [14] } \\
\text { (ionization effects) } \\
\text { (non-ionizing) }\end{array}$ & $6-30$ & $\begin{array}{l}\text { Either few } \\
\text { MeV or } \\
\text { broad }\end{array}$ & $\begin{array}{l}10^{4}-10^{5} \\
10^{4}-10^{5}\end{array}$ \\
\hline Materials damage [15] & $6-30$ & Few MeV & $10^{4}-10^{6}$ \\
\hline Radiation Biology [16] & $6-30$ & Few MeV & $10^{4}-10^{6}$ \\
\hline
\end{tabular}

\subsection{Neutron production by deuteron breakup}

Deuterons are very weakly bound, with a Q-value of 2.22 MeV. The breakup of a deuteron in the Coulombic field of a heavier nucleus is a basic nuclear physics problem that has been studied extensively since first suggested by Oppenheimer and Phillips in 1935 [17]. Assuming pure Coulombic breakup in its most simplistic manifestation, the deuteron deaccelerates as it approaches the heavy target nucleus. At a radius $R_{B}$, it breaks up, and the proton and neutron each take half of the available energy at that time. The proton reaccelerates on the way out, leaving the neutron with an energy given by

$$
E_{n}=1 / 2\left(E_{d}-Z^{2} / R_{B}-2.22\right) M e V
$$

This energy is well defined, so theoretically this process should yield a forward focused neutron with an energy spread determined by the width of the breakup radius.

Several early experiments [18-20] studied the breakup process at low energies $\left(E_{d}=8-17 \mathrm{MeV}\right)$ on a range of heavy targets. By measuring the energy and angle of the proton in coincidence with neutrons, it was determined that breakup occurred well outside of the nuclear radius. This body of work gave evidence that Coulombic breakup dominated at forward angles for heavier targets, but nuclear breakup played a role at all energies for lighter targets like $\mathrm{Be}$ and $\mathrm{C}$, and became more important for all targets as the deuteron energy was raised.

In contrast to the early experiments, a more recent experiment by Okamura et al. [21] measured breakup at very forward angles at $56 \mathrm{MeV}$. They concluded that the nuclear breakup was more dominant for the heaviest targets. This data has been validated by modern 3-body theories [22]; however, these models have not yet been demonstrated to work at lower energies. The role of Coulombic and nuclear breakup is clearly changing in this energy region and systematic measurements are needed.

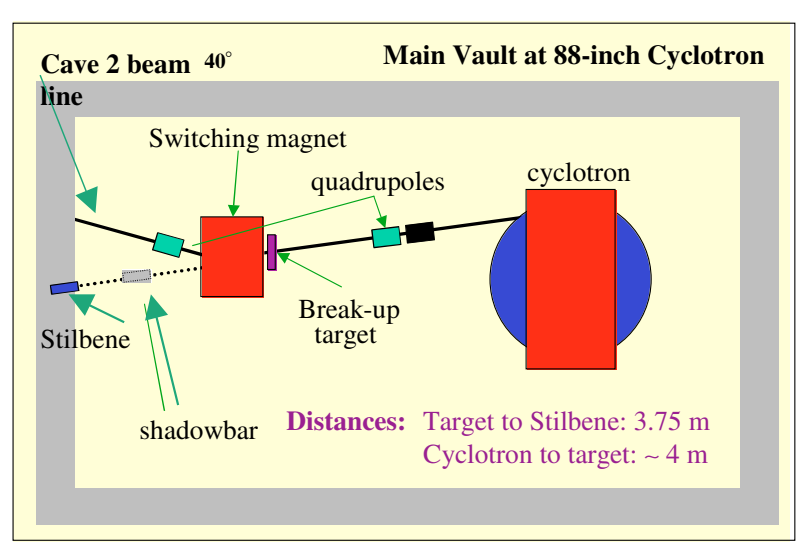

Fig. 1. Experimental Setup. The neutron production target for thin targets is located in the Vault before the main switching magnet, which diverts the unreacted beam into Cave 2. Neutron time of flight is measured 3.75 meter downstream with a Stilbene or NE213 detector inside the shielding. For thick target neutron production, the target can be located in either Cave 2 or the adjacent Cave 3 .

\section{Experimental method}

A schematic of the neutron beamline is shown in figure 1. Two target stations are available. Thin target "quasi-monenergetic" neutrons are available in the Cyclotron Vault for offline cross section measurements on either stable or radioactive targets. The deuteron beam is dumped well downstream in Cave 2. Thick target "broad energy" neutrons are available at an irradiation station in Cave 2.

The absolute flux is measured at either target station using standard activation foil techniques [23]. By using a variety of activation foils and measuring reaction products with different thresholds, some energy information can be obtained as well. This technique utilizes the code SAN-II [24]. In particular, by comparing results from reaction products that are sensitive to thermal neutrons, the background from thermals can be estimated.

The energy distributions can be measured directly with a proton-recoil detector by using neutron time-of-flight (TOF) combined with pulse shape discrimination to distinguish the neutrons from the gamma-rays. This setup has been described in a previous paper [25].

In order to perform neutron time-of-flight measurements, it is necessary to tune the Cyclotron to a narrow phase width optimally less than 2 nsec. This is complicated because the 88Inch Cyclotron does not have single turn extraction and the natural phase width of its beams is $5-10$ nsec. A fast Faraday cup was developed in the 1990s as a diagnostic device used to tune a set of slit collimators inserted into the center region of the Cyclotron. The slits intercept a single orbit and produce extracted beams with phase widths of 1-2 nsec [26]. The fast Faraday cup and slit collimators have been reconstituted and moved to the Cyclotron Vault in order to make the neutron TOF measurements easier.

\section{Results}

Initial characterization of the neutron spectra and fluxes with thin targets have been done at $29 \mathrm{MeV}$ and $20 \mathrm{MeV}$, 


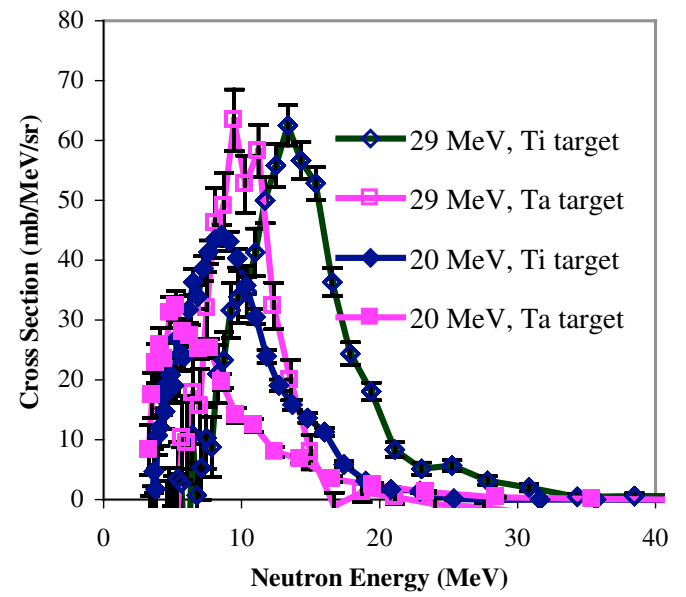

Fig. 2. Typical neutron energy spectra at $0^{\circ}$ for Ti and Ta targets.

Table 2. Neutrons from deuteron breakup at $0^{\circ}$.

\begin{tabular}{cccccc}
\hline Target & $\begin{array}{c}\mathrm{E}_{\mathrm{d}} \\
(\mathrm{MeV})\end{array}$ & $\begin{array}{c}\left\langle\mathrm{E}_{\mathrm{n}}\right\rangle \\
(\mathrm{MeV})\end{array}$ & $\begin{array}{c}\text { FWHM } \\
(\mathrm{MeV})\end{array}$ & $\begin{array}{c}\text { Fluence } \\
\text { rate } \\
(\mathrm{n} / \mathrm{sec} / \\
\left.\mathrm{cm}^{2} / \mu \mathrm{A}\right)\end{array}$ & $\begin{array}{c}\text { Fluence } \\
\text { rate* } \\
(\mathrm{n} / \mathrm{sec} / \\
\left.\mathrm{cm}^{2} / \mu \mathrm{A}\right)\end{array}$ \\
\hline $\mathrm{Ti}$ & 20 & 8.7 & 7.0 & $2 \times 10^{4}$ & $8.5 \times 10^{4}$ \\
$\mathrm{Ti}$ & 29 & 13.6 & 9.0 & $7 \times 10^{4}$ & - \\
$\mathrm{Ta}$ & 20 & 6.0 & 6.0 & $2 \times 10^{4}$ & - \\
$\mathrm{Ta}$ & 29 & 10.3 & 5.5 & $5 \times 10^{4}$ & - \\
\hline
\end{tabular}

rough extrapolation from TOF data, 1 meter from target.

* measured

chosen because the first applications under consideration for stewardship applications and for radiation effects - require neutron spectra peaked in the $10-14 \mathrm{MeV}$ range. Neutron energy distributions have been measured using thin targets for the following target/energy combinations: Ti and Ta at 20 and $29 \mathrm{MeV}$. Typical energy spectra for the cases of $\mathrm{Ti}$ and $\mathrm{Ta}$ targets at $0^{\circ}$ are given in figure 2 . Additional measurements were taken using Ta targets, examining the effect of target thickness and measuring angular distributions.

With the current target geometry, activation foil measurements have been completed with the Ti target at $20 \mathrm{MeV}$. Table 3 summarizes the present data for the two targets at 20 and $29 \mathrm{MeV}$, giving neutron energy, FWHM of the energy distribution and flux as estimated from the energy measurements or as measured directly using the activation method. The uncertainty in the extracted mean neutron energy and FWHM is $0.3 \mathrm{MeV}$ in all cases.

Neutron spectra for deuterons stopping in Be targets have been measured at several angles for $E_{d} \leq 20 \mathrm{MeV}$. The thick target distributions using Be targets at $E_{d}=20 \mathrm{MeV}$ agree with data taken at other facilities $[9,27]$ and will not be presented here.

\section{Discussion}

There are several points that can be deduced from the results of table 3 and figure 2. Firstly, the mean energy is strongly dependent on target, decreasing significantly between $\mathrm{Ti}$ and
Ta at both energies. This is in contrast with the data at $56 \mathrm{MeV}$ [21], that showed an almost constant - albeit double-peaked - mean energy for targets of $\mathrm{C}, \mathrm{Ca}, \mathrm{Zr}$ and $\mathrm{Pb}$. In that work, the major effect of target mass was the disappearance of the lower of the two energy peaks for $\mathrm{Pb}$. The target effect is also not reproduced by preliminary calculations based on modern coupled channel calculations [28].

For both targets, the neutron FWHMs are wider than expected from pure Coulombic breakup and from what was seen at the higher energies of ref. [21]. This may be partially due to the large neutron background subtraction that must be done because of the experimental location in the Cyclotron vault. In particular, a large number of neutrons are produced at the deflectors at the exit of the Cyclotron, and this source of neutrons cannot be corrected for by using a shadowbar [25]. In spite of this, the width is somewhat narrower for Ta than it is for Ti targets, indicating that effect is not totally due to background subtraction.

It can be seen from figure 2 that the error bars are significantly larger for the Ta target than for Ti, especially at $20 \mathrm{MeV}$. This is because the beam scattering in the target is greater for the heavier target, causing beam scraping downstream and an increase in the background that must be subtracted in order to obtain the energy distribution. This is a fundamental limitation on the method.

The flux is relatively constant between the two targets at both energies, and increases with energy. This agrees roughly with the results of ref. [21] at $56 \mathrm{MeV}$, in which the authors examined the dependence of the energy-integrated cross section on target atomic number. In that work, comparisons with DWBA calculations led them to conclude the presence of a large contribution from nuclear breakup at forward angles for the $\mathrm{Pb}$ target. This has yet to be confirmed in our results at lower energies.

\section{Conclusions}

We have recently begun a set of measurements to systematically explore the energy and angle dependence of the neutron production during the breakup process in order to understand the disagreements between our measurements at 20 and $29 \mathrm{MeV}$ and the work of Okamura et al. [11] at $56 \mathrm{MeV}$, and the discrepancies between our measurements and coupled channel calculations.

At the same time, the shielding around Cave 2 is being enhanced to allow operation at deuteron intensities greater than $1 \mu \mathrm{A}$. When this is complete, absolute flux measurements will be completed for both targets at several energies, and the first cross section measurement on a radioactive target will take place.

Future plans include installation of a magnet in Cave 2 so that the thin production target can be moved out of the Vault; this will eliminate the largest source of neutron background, from scraping on the deflectors at the exit to the Cyclotron.

This work was supported by the US Department of Energy Office of Science Contract No. DE-AC02-05CH11231 and the US Department of Energy National Nuclear Security Administration Contract No. DE-FG03-03NA00078, and under the auspices of the US Department 
of Energy by the University of California, Lawrence Livermore National Laboratory under contract No. W-7405-Eng-48.

\section{References}

1. F.P. Brady, J.L. Romero, Nucl. Sci. Eng. 106, 318 (1990).

2. H. Conde, S. Hultqvist, N. Olsson, T. Ronnqvist, R. Zorro, J. Blomgren, G. Tibell, A. Hakansson, O. Jonsson, A. Lindholm, L. Nilsson, P.U. Renberg, A. Brockstedt, P. Elkstrom, M. Osterlund, F.P. Brady, Z. Szeflinski, Nucl. Instrum. Meth. A 292, 121 (1990).

3. H. Schuhmacher, H.J. Brede, V. Dangendorf, M. Kuhfuss, J.P. Meulders, W.D. Newhauser, R. Nolte, Nucl. Instrum. Meth. A 421, 284 (1999).

4. T. Nakamura, M. Takada, N. Nakao, M. Baba, T. Iwasaki, H. Nakashima, S. Tanaka, S. Meigo, Y. Sakamoto, Y. Nakane, S. Tanaka, Y. Uwamino, N. Nakanishi, in Proc. of the Intl. Conf. On Nuclear Data for Science and Technology, Conf. Series of the Italian Physical Society, Bologna, 1997, p. 1508.

5. W.R. McMurray, D.G. Aschman, K. Bharuth-Ram, R.W. Fearick, Nucl. Instrum. Meth. A 329, 217 (1993).

6. R.C. Byrd, W.C. Sailor, Nucl. Instrum. Meth. A 274, 494 (1989).

7. S.N. Abramovich, B.Ja. Guzhovskij, V.A. Zherebcov, A.G. Zvenigorodskij, Vop. At. Nauki i Tekhn. 17 (1984).

8. S. Brandenburg, J.P.M. Beijers, F. Clapier, H. Laurent, L. Lebreton, M. Mirea, N. Pauwels, M.-G. Saint-Laurent, R.G.T. Zegers, KVI Annual Report, 1999.

9. J.P. Meulders, P. Leleux, P.C. Macq, C. Pirart, Phys. Med. Biol. 20, 235 (1975).

10. M.J. Saltmarsh, C.A. Ludemann, C.B. Fulmer, R.C. Styles, Nucl. Instrum. Meth. 145, 81 (1977).

11. L. Ahle, LLNL (private communications).
12. D.R. Welch, H. Kislev, G.H. Miley, Rev. Sci. Instrum. 59, 610 (1988).

13. G. Aliberti, G. Palmiotti, M. Salvatores, T.K. Kim, T.A. Taiwo, M. Anitescu, I. Kodeli, E. Sartori, J.C. Bosq, J. Tommasi, Ann. Nucl. Energy 33, 700 (2006).

14. H. Braunig, F. Wulf, Radiat. Phys. Chem. 43, 105 (1994).

15. D. Steiner, Nucl. Sci. Eng. 58, 107 (1975).

16. T. Ohnishi, A. Takahashi, K. Ohnishi, J. Radiat. Res. 43, 7 (2002).

17. J.R. Oppenheimer, M. Phillips, Phys. Rev. 48, 500 (1935).

18. E.C. May, B.L. Cohen, T.M. O'Keefe, Phys. Rev. 164, 1253 (1967).

19. C.L. Fink, B.L. Cohen, J.C. Van der Weerd, R.J. Petty, Phys. Rev. 185, 1568 (1969).

20. L. Jarczyk, J. Lang, R. Muller, D. Balzar, P. Viatte, P. Pramier, Phys. Rev. C 8, 68 (1973).

21. H. Okamura, S. Hatori, N. Matsuoka, T. Noro, A. Okihana, H. Sakai, H.M. Shimizu, K. Takeshita, T. Yamaya, Phys. Lett. B 325, 308 (1994).

22. J.A. Tostevin, S. Rugmai, R.C. Johnson, Phys. Rev. C 57, 3225 (1998).

23. William S. Lyon Jr. (ed.), Guide to Activation Analysis, D. Van Nostrand Company, 1964.

24. P.J. Griffin et al., User's Manual for SNL SAND-II, SNL-SND93-3957 (April 1994).

25. D.L. Bleuel, M.A. McMahan, L. Ahle, B.R. Barquest, J. Cerny, L.H. Heilbronn, C.C. Jewett, in Proc of the Conf. on Appl. of Accel. in Research and Industry, 2006, Nucl. Instrum. Meth. B (in press).

26. D.J. Clark, X.Q. Xie, M.A. McMahan, Lawrence Berkeley National Lab Internal Report, LBNL-44339 (1999).

27. J. Collot, P. De Saintignon, P. Gabor, A. Hoummada, G. Mahout, D. Marchand, F. Merchez, E. Leon Florian, C. Leroy, Ph. Jean, B. Merkel, Nucl. Instrum. Meth. A 350, 525 (1994).

28. I. Thompson, LLNL (private communication). 